\title{
Efficacy and Safety of Dulaglutide by Baseline HbA1c in Chinese Patients with Type 2 Diabetes: A Post Hoc Analysis
}

\author{
Miao Yu - Guo Yue Yuan · Bin Zhang $\cdot$ Hai Ya Wu $\cdot$ Xiao Feng Lv
}

Received: September 19, 2019 / Published online: April 10, 2020

(C) The Author(s) 2020

\section{ABSTRACT}

Introduction: To evaluate the efficacy and safety of dulaglutide 0.75 and $1.5 \mathrm{mg}$ in patients with type 2 diabetes mellitus (T2DM) by baseline glycated hemoglobin $(\mathrm{HbA} 1 \mathrm{c})<8.5 \%$ or $\geq 8.5 \%$ after 26 weeks of treatment.

Enhanced Digital Features To view enhanced digital features for this article go to https://doi.org/10.6084/ m9.figshare.11439636.

Electronic supplementary material The online version of this article (https://doi.org/10.1007/s13300020-00804-2) contains supplementary material, which is available to authorized users.

M. Yu

Department of Endocrinology, Key Laboratory of Endocrinology, National Health Commission, Peking Union Medical College Hospital, Chinese Academy of Medical Sciences, Peking Union Medical College, Beijing 100730, China e-mail:yumiaoxh@163.com

\section{G. Y. Yuan}

Department of Endocrinology, Affiliated Hospital of Jiangsu University, Jiangsu, China

B. Zhang · H. Y. Wu (ه)

Medical Department, Lilly Suzhou Pharmaceutical Co. Ltd, Shanghai, China

e-mail: wu_hai_ya@hotmail.com

\section{F. Lv (ه)}

Department of Endocrinology, The Seventh Medical Center of PLA General Hospital, Beijing, China e-mail: neifenmike@126.com
Methods: Assessment of the Weekly AdministRation of dulaglutide in Diabetes (AWARD) China 1 (CHN1) study (NCT01644500, $n=556$ ) included patients on dulaglutide vs. glimepiride who were treatment naïve or on monotherapy but discontinued therapy. AWARD-CHN2 (NCT01 $648582, n=591$ ) patients were on dulaglutide vs. insulin glargine and continued on metformin and/or sulfonylurea. Mean daily dose of glimepiride and insulin glargine was $2.51 \mathrm{mg}$ and $21.0 \mathrm{IU}$, respectively. Post hoc analyses were conducted based on mixed-model repeated measures using a modified intent-to-treat analysis set with only the Chinese population. Change from baseline in HbA1c and body weight was analyzed by individual study.

Results: In the two studies, $70.1 \%$ of patients in AWARD-CHN1 and 59.7\% in AWARD-CHN2 had baseline HbA1c < 8.5\% (mean HbA1c 7.4\% and 7.6\%, respectively) and $29.9 \%$ in AWARD-CHN1 and $40.3 \%$ in AWARD-CHN2 had baseline $\mathrm{HbA} 1 \mathrm{c} \geq 8.5 \%$ (mean HbA1c 9.2\% and 9.4\%, respectively). In AWARD-CHN1, the HbA1c reductions at 26 weeks with baseline $\mathrm{HbA1c}<$ $8.5 \%$ and $\geq 8.5 \%$, respectively, were dulaglutide $1.5 \mathrm{mg}$ : $-1.1 \%$ and $-2.2 \%$; dulaglutide $0.75 \mathrm{mg}$ : $-0.9 \%$ and $-2.0 \%$; glimepiride: $-0.7 \%$ and - $1.4 \%$. In AWARD-CHN2, the HbA1c reductions at 26 weeks with baseline HbA1c $<8.5 \%$ and $\geq 8.5 \%$, respectively, were dulaglutide $1.5 \mathrm{mg}$ : $-1.2 \%$ and $-2.3 \%$; dulaglutide $0.75 \mathrm{mg}:-1.0 \%$ and $-1.7 \%$; and insulin glargine: $-0.6 \%$ and $-1.7 \%$. Irrespective of baseline HbA1c, body 
weight decreased with both dulaglutide doses and increased with either glimepiride or insulin glargine at 26 weeks. Dulaglutide demonstrated low incidence of hypoglycemia in both doses in the two trials. Hypoglycemia incidence was generally lower in patients with baseline HbA1c $\geq 8.5 \%$.

Conclusions: Dulaglutide demonstrated significantly greater $\mathrm{HbA1c}$ reduction with weight loss and lower risk of hypoglycemia compared with active comparators in Chinese patients with T2DM irrespective of baseline HbA1c, with much greater $\mathrm{HbA1c}$ reductions in patients with a higher baseline HbA1c.

Trial registration: ClinicalTrials.gov identifier, NCT01644500 and NCT01648582.

Keywords: Baseline HbA1c; Dulaglutide; Glimepiride; Hypoglycemia; Insulin glargine; Type 2 diabetes mellitus

\section{Key Summary Points}

\section{Why carry out this study?}

Efficacy and safety of dulaglutide were studied in two phase III randomized trials (AWARD-CHN1 and AWARD-CHN2 study) in Chinese adult patients with type 2 diabetes mellitus (T2DM), which demonstrated significant glycated hemoglobin $(\mathrm{HbA1c})$ reduction.

The effects of dulaglutide in Chinese patients with T2DM and different baseline HbA1c levels $(<8.5 \%$ and $\geq 8.5 \%)$ have not yet been reported.

Thus, the present post hoc analysis of two phase III randomized trials is designed to evaluate the efficacy and safety of dulaglutide $1.5 \mathrm{mg}$ and dulaglutide $0.75 \mathrm{mg}$ in Chinese patients with T2DM by baseline HbA1c $(<8.5 \%$ and $\geq 8.5 \%)$ after 26 weeks of treatment. Also, the present post hoc analysis helps in understanding the efficacy and safety of dulaglutide in patients with T2DM and a higher HbA1c level.

\section{What was learned from the study?}

In the present post hoc analysis of two studies, dulaglutide demonstrated significantly greater $\mathrm{HbA} 1 \mathrm{c}$ reduction with greater weight loss and less hypoglycemia in Chinese patients with T2DM irrespective of baseline HbA1c, with greater $\mathrm{HbA1c}$ reductions in patients with a higher baseline HbA1c.

Dulaglutide was well tolerated, with a safety profile similar to other GLP-1RAs.

\section{INTRODUCTION}

Type 2 diabetes mellitus (T2DM) is the most common form of diabetes and a chronic metabolic disorder with increasing worldwide prevalence [1]. Insulin resistance and a progressive decline in $\beta$-cell function [2] characterize it. China has the world's largest diabetes epidemic, with the estimated overall prevalence of $10.9 \%$ among adults as reported in a survey conducted in 2013 [3].

In China, patients with T2DM have poor glycemic control prior to the initiation of insulin [4]. According to the 2017 Chinese Diabetes Society guidance, insulin therapy is recommended in patients with T2DM when lifestyle modifications and oral antihyperglycemic medications (OAM) fail to achieve adequate glycated hemoglobin (HbA1c) $<7 \%$ [5]. However, in Chinese clinical practice, realworld observational studies $[4,6]$ have demonstrated that insulin treatment is only initiated when HbA1c is about $9 \%$ and the rate of achieving glycemic control is relatively low. In addition, hypoglycemia [7] and weight increase [8] associated with insulin therapy remain barriers to overcome. Patient-centered diabetes management must balance the benefits of glycemic control and potential weight effects against the risk of adverse events (AEs), particularly hypoglycemia and weight gain [9]. Unlike therapy with insulin and sulfonylureas, glucagon-like peptide-1 receptor agonists (GLP-1RAs) 
stimulate insulin secretion in a glucose-dependent pattern, improving glucose control with weight loss and a lower risk of hypoglycemia [10]. Several lines of clinical evidences suggest a better risk-to-benefit ratio of GLP-1RAs compared with traditional antidiabetic drugs, such as glimepiride, which is widely used across East Asia. GLP-1RAs are generally well tolerated, although gastrointestinal (GI) AEs are commonly observed across the class [11-14].

Dulaglutide, a long-acting glucagon-like peptide-1 receptor agonist, was approved in 2014 for the treatment of T2DM [12]. Dulaglutide has been evaluated across the diabetes treatment continuum in the Assessment of Weekly AdministRation of LY2189265 in Diabetes (AWARD) trials in mainly Caucasians patients with T2DM [9]. In AWARD studies, dulaglutide demonstrated significant improvements in glycemic control irrespective of gender, duration of diabetes $(<5, \geq 5$ years and $<10, \geq 10$ years) or baseline HbA1c $(<8.5 \%, \geq 8.5 \%)$, with greater HbA1c and fasting blood glucose (FBG) reductions in patients with a higher baseline HbA1c [9]. Moreover, lower risk of weight gain or hypoglycemia was observed with dulaglutide compared with active comparators such as metformin, sitagliptin, exenatide twice daily, and insulin glargine. Efficacy and safety of dulaglutide were also studied in two phase III randomized trials (AWARD-China 1 [CHN1] and AWARD-CHN2 study) in Chinese adult patients with T2DM, which demonstrated significant HbA1c reduction $[11,12]$. However, the effects of dulaglutide in Chinese patients with T2DM and different baseline HbA1c levels $(<8.5 \%$ and $\geq 8.5 \%$ ) have not yet been reported. Hence, this post hoc analysis of two trials aimed to evaluate the efficacy and safety of dulaglutide $1.5 \mathrm{mg}$ and $0.75 \mathrm{mg}$ in Chinese patients with T2DM with a baseline HbA1c $(<8.5 \%$ and $\geq 8.5 \%)$ after 26 weeks of treatment.

\section{METHODS}

\section{Study Design and Patients}

The present analysis included patients from two randomized, phase III clinical trials from the
AWARD program, AWARD-CHN1 (NCT016 44500) and AWARD-CHN2 (NCT01648582). The primary end point of both studies was to assess the change in HbA1c from baseline at week 26 . Individual trial results were previously published [11, 12]. In AWARD-CHN1, the enrolled patients were treatment naïve or on monotherapy but discontinued therapy. In AWARD-CHN2, the enrolled patients were on metformin with or without sulfonylurea. Both studies assessed the efficacy and safety of once weekly dulaglutide (1.5 mg and $0.75 \mathrm{mg}$ ) vs. active comparators [glimepiride (GLIM) in AWARD-CHN1 and glargine (GLAR) in AWARD-CHN2] in Chinese adult patients with T2DM. Institutional ethics committee approval was obtained for the studies, and written informed consent was taken from each patient before participation. Both studies were conducted in consensus with the Declaration of Helsinki, good clinical practice guidelines, and applicable laws and regulations.

\section{End Points and Statistical Analysis}

The primary end point of this analysis was conducted based on a modified intent-to-treat analysis set with only the Chinese population, consisting of all randomized patients who had a baseline HbA1c measurement with at least one post-baseline $\mathrm{HbA1c}$ measurement and received at least one dose of study drug. All efficacy and safety analyses were conducted at 26 weeks, as this was a common primary efficacy time point for both AWARD-CHN studies. Efficacy analyses of changes from baseline in HbA1c and FBG, proportion of patients achieving HbA1c targets of $<7 \%$ and $\leq 6.5 \%$, and self-monitored blood glucose (SMBG) level at baseline and at week 26 after dulaglutide $0.75 \mathrm{mg}$ and $1.5 \mathrm{mg}$ treatments were analyzed by individual study. Safety end points included change in body weight, incidence and rate of all hypoglycemia (total hypoglycemia, plasma glucose $\leq 3.9 \mathrm{mmol} / \mathrm{l}$ ), severe hypoglycemia episodes (defined as requiring treatment), nocturnal hypoglycemia, and documented symptomatic hypoglycemia, which were summarized by individual study. In addition, GI AEs (occurring in $\geq 5 \%$ of patients) 


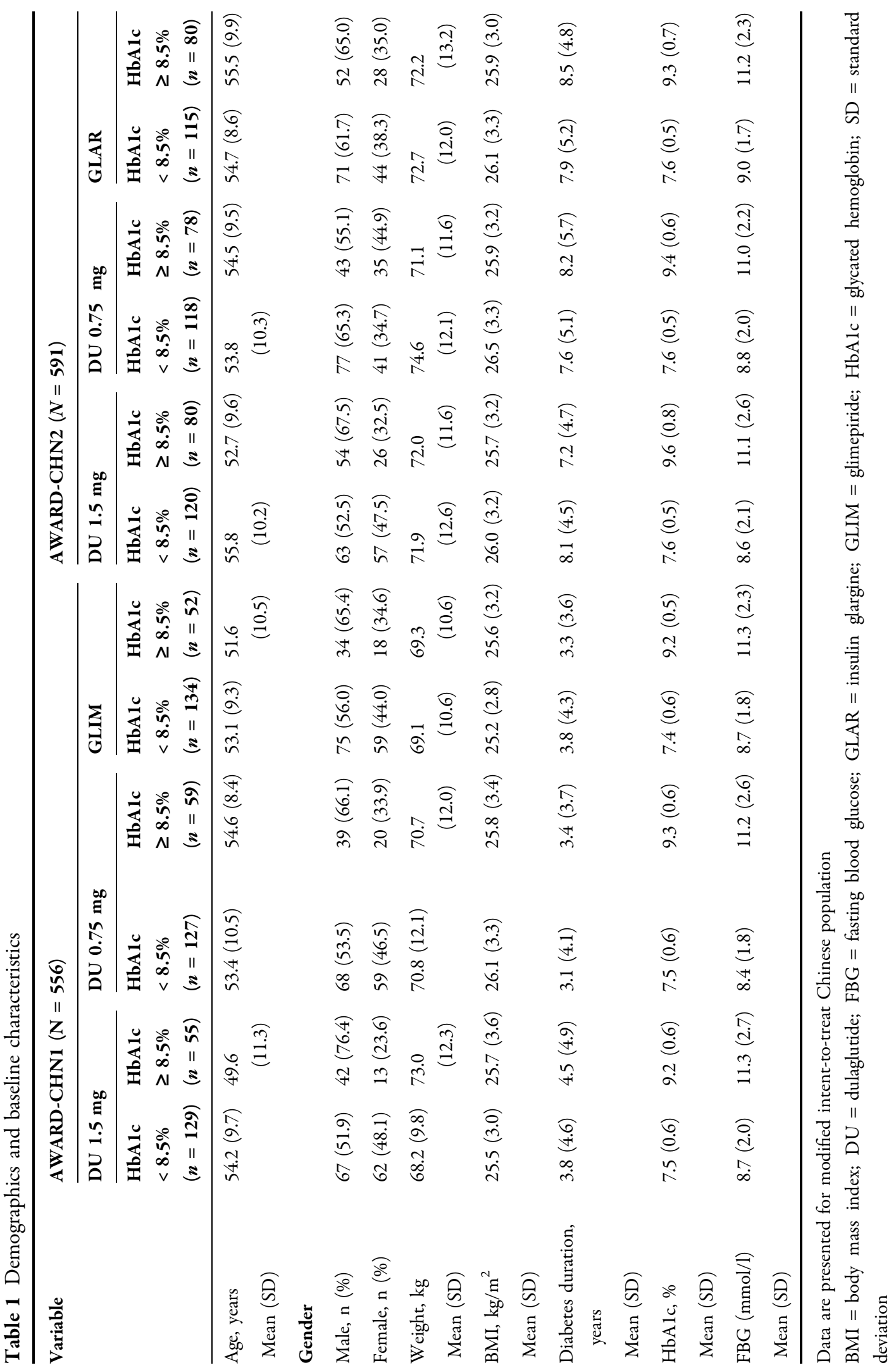




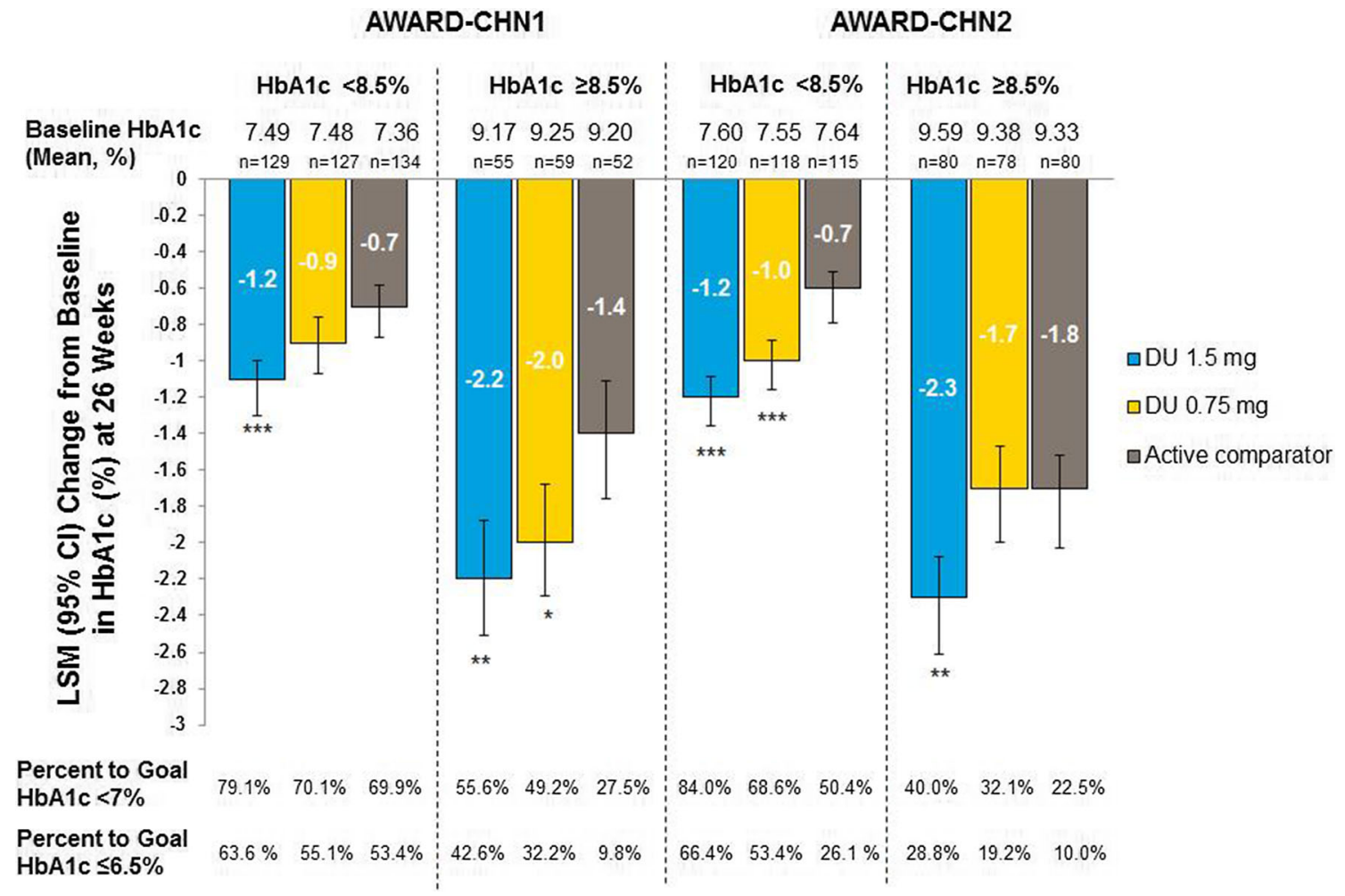

Fig. $1 \mathrm{HbAlc}$ reduction at week 26 with baseline HbAlc $<8.5 \%$ and $\geq 8.5 \% .{ }^{*} p<0.05,{ }^{* *} p<0.01,{ }^{* * *} p<0.001$, dulaglutide vs. active comparator. $D U$ dulaglutide, HbAlc glycated hemoglobin, $L S M$ least-squares mean

were summarized by individual study. Changes from baseline in $\mathrm{HbA1c}$, FBG, SMBG, and weight were analyzed using mixed-model repeated measures with model terms including treatment group, OAM stratum, visits, treatment by visit interaction, and baseline value. Least squares (LS) means and corresponding 95\% confidence intervals (CIs) are presented. All analyses were conducted using SAS 9.2 (SAS Institute, Inc., Cary, NC, USA).

\section{RESULTS}

\section{Patient Disposition and Baseline Characteristics}

In the AWARD-CHN1 study, 70.1\% (390/556) of patients had baseline HbA1c $<8.5 \%$ and $29.9 \%$ $(166 / 556)$ had baseline HbA1c $\geq 8.5 \%$, whereas $59.7 \%(353 / 591)$ of patients in the AWARDCHN2 study had baseline HbA1c $<8.5 \%$ and $40.3 \%$ (238/591) had baseline HbA1c $\geq 8.5 \%$.
The demographics and baseline characteristics of all 1147 patients were stratified by baseline HbA1c and are listed in Table 1. Overall, patient characteristics were comparable between the treatment groups for each subgroup. Patients with higher baseline HbA1c $(\geq 8.5 \%)$ had higher mean FBG than patients with lower baseline HbA1c $(<8.5 \%)$.

\section{Efficacy}

In both HbA1c subgroups, dulaglutide $1.5 \mathrm{mg}$ and $0.75 \mathrm{mg}$ treatments resulted in significantly greater $\mathrm{HbA1c}$ reductions compared with active comparators, with much greater $\mathrm{HbA1c}$ reductions observed for patients with $\mathrm{HbA} 1 \mathrm{c} \geq 8.5 \%$ than for patients with HbA1c $<8.5 \%$ (Fig. 1). Moreover, dulaglutide $1.5 \mathrm{mg}$ and $0.75 \mathrm{mg}$ treatments resulted in a greater proportion of patients achieving HbA1c values of less than both $6.5 \%$ and $7 \%$ at 26 weeks compared with active comparators (GLIM/GLAR) in both the 


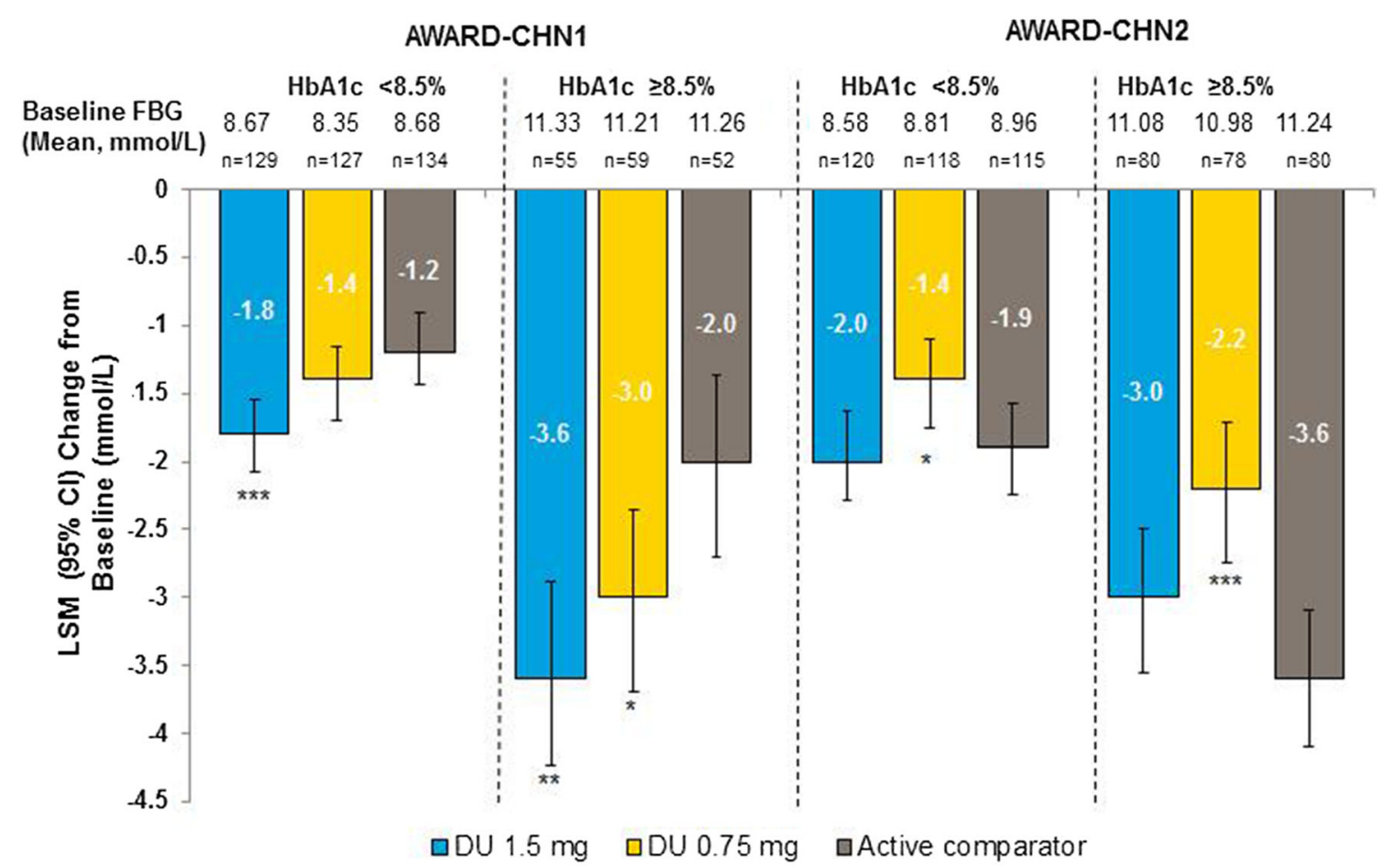

Fig. 2 Change in fasting blood glucose from baseline to week $26 .{ }^{*} p<0.05,{ }^{* *} p<0.01,{ }^{* * *} p<0.001$, dulaglutide vs. active comparator. $D U$ dulaglutide, FBG fasting blood glucose, HbAlc glycated hemoglobin, LSM least squares mean

studies (AWARD-CHN1 and AWARD-CHN2) (Fig. 1). Also, in both studies, the HbA1c $<8.5 \%$ subgroup had a greater proportion of patients achieving HbA1c values of less than both $6.5 \%$ and $7 \%$ at 26 weeks compared with the $\mathrm{HbA1c} \geq 8.5 \%$ subgroup. Consistent with HbA1c reduction, the FBG reductions ( $\mathrm{mmol} / \mathrm{l}$ ) from baseline were greater in patients with $\geq 8.5 \%$ than in patients with HbA1c $<8.5 \%$ for both dulaglutide $1.5 \mathrm{mg}$ and $0.75 \mathrm{mg}$ treatments at 26 weeks compared with active comparators (GLIM/GLAR) (Fig. 2). Mean 7-point SMBG profiles by treatment groups at baseline and week 26 are shown in Fig. 3 for patients with $\mathrm{HbA} 1 \mathrm{c}<8.5 \%$ and $\mathrm{HbA} 1 \mathrm{c} \geq 8.5 \%$, respectively. In the $\mathrm{HbA} 1 \mathrm{c}<8.5 \%$ and $\mathrm{HbA1c}$ $\geq 8.5 \%$ subgroups, dulaglutide demonstrated greater reduction in SMBG values from baseline after 26 weeks compared with active comparators (GLIM/GLAR) for all time points, except morning pre-meal (Fig. 3, Supplementary Table S1). Dulaglutide achieved a similar effect on weight in patients with $\mathrm{HbA1c} \geq 8.5 \%$ and those with $<8.5 \%$ (Fig. 4). In AWARD-CHN1, for both subgroups, LS mean body weight was decreased from baseline in dulaglutide-treated patients and increased from baseline in glimepiride-treated patients after 26 weeks (Fig. 4). In AWARD-CHN2, for both subgroups, LS mean body weight was decreased from baseline in dulaglutide-treated patients and increased from baseline in insulin glargine-treated patients after 26 weeks (Fig. 4). For both subgroups, the differences in change in body weight between the treatment groups (dulaglutide vs. GLIM/ GLAR) were statistically significant $(p<0.001)$.

\section{Safety}

The incidence and rate of hypoglycemia (symptomatic, nocturnal, and severe) events in AWARD-CHN1 and AWARD-CHN2 by HbA1c levels ( $<8.5 \%$ vs. $\geq 8.5 \%$ ) are shown in Table 2 . In AWARD-CHN1 and AWARD-CHN2, dulaglutide demonstrated a low incidence of hypoglycemia in both doses. Hypoglycemia 


\section{a AWARD-CHN1 $<8.5 \%$}

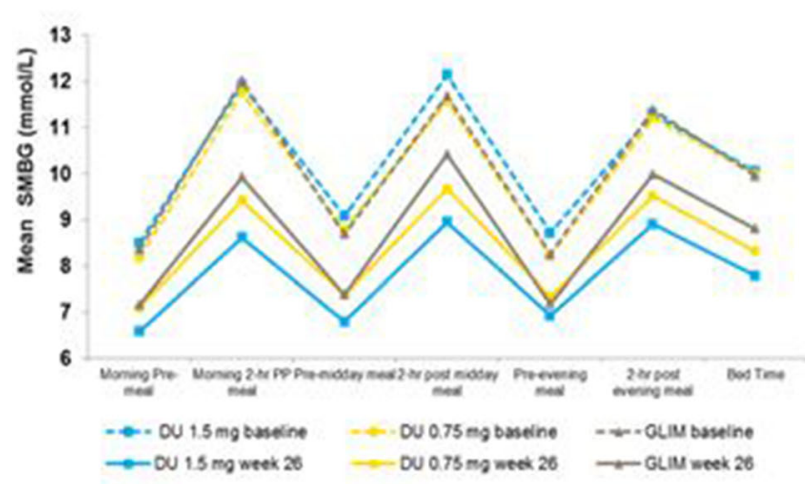

C AWARD - CHN2 $<8.5 \%$

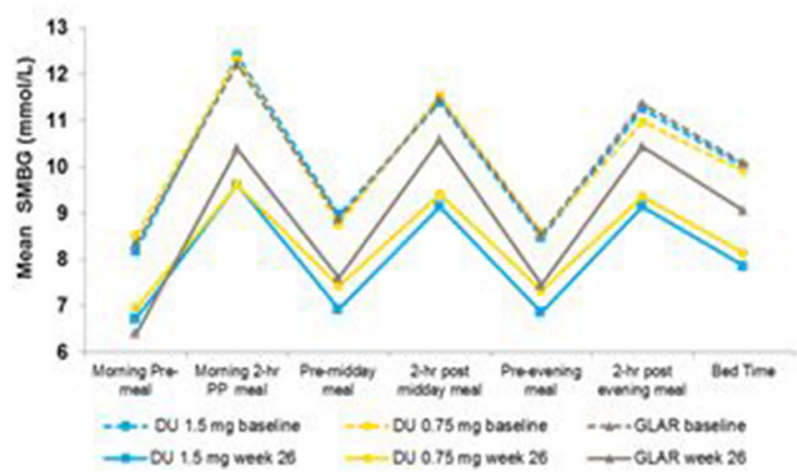

b AWARD-CHN $128.5 \%$

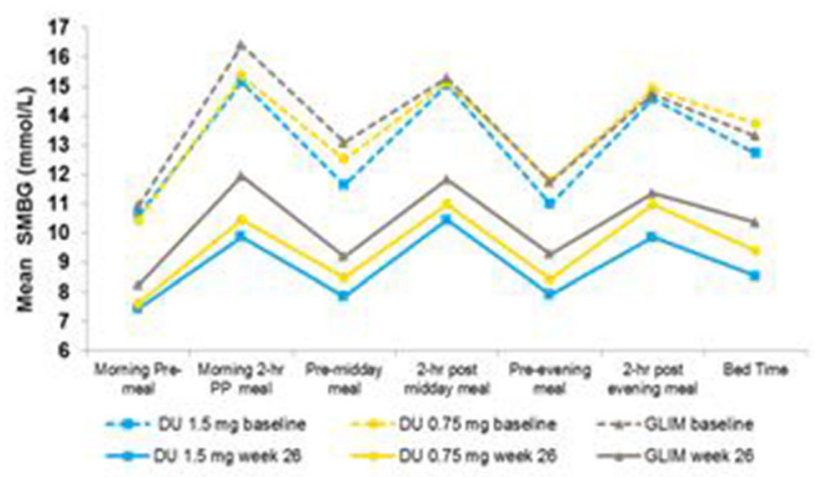

d AWARD-CHN2 $28.5 \%$

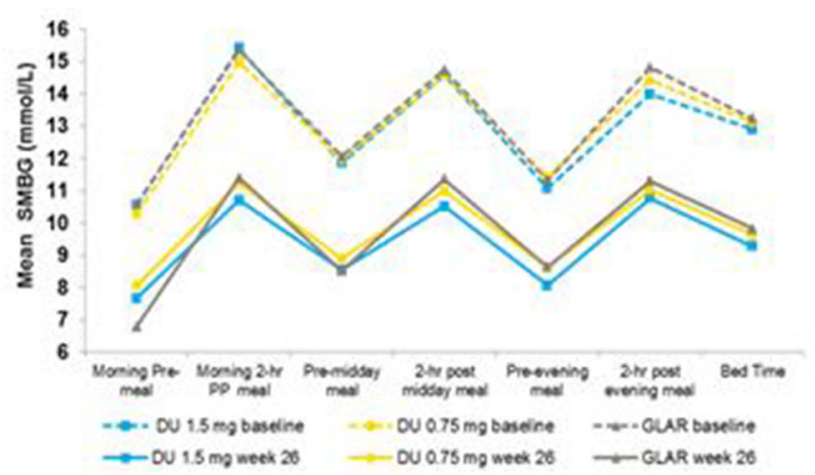

Fig. 3 Seven-point self-monitored blood glucose (smbg) profiles by time of day. DU dulaglutide, GLAR glargine, GLIM glimepiride, $P P$ postprandial

incidence was generally lower in patients with baseline HbA1c $\geq 8.5 \%$. The incidences of total, symptomatic, and nocturnal hypoglycemia were considerably less frequent with dulaglutide than with GLIM/GLAR in both subgroups (Table 2). In patients with baseline HbA1c $\geq 8.5 \%$, the incidence of total, symptomatic, and nocturnal hypoglycemia was generally lower than in patients with baseline HbA1c $<$ $8.5 \%$ in either treatment group. Severe hypoglycemia was not reported in any of the subgroups. In AWARD-CHN1 and AWARD-CHN2, GI AEs occurred more frequently with dulaglutide than GLIM/GLAR in both subgroups. Dulaglutide-treated patients with $\mathrm{HbA} 1 \mathrm{c}<$ $8.5 \%$ had higher incidences of GI treatmentemergent AEs (TEAEs) such as diarrhea, nausea, vomiting, abdominal distension, and upper abdominal pain compared with the patients with $\mathrm{HbA} 1 \mathrm{c} \geq 8.5 \%$ in either treatment arm (Table 3).

\section{DISCUSSION}

This is the first analysis designed to explore the efficacy and safety of once weekly dulaglutide $1.5 \mathrm{mg}$ and $0.75 \mathrm{mg}$ compared with glimepiride or insulin glargine by subgroups of HbA1c $(<8.5 \%$ or $\geq 8.5 \%)$ in Chinese patients with T2DM. In this subgroup analysis, dulaglutide $1.5 \mathrm{mg}$ and $0.75 \mathrm{mg}$ treatments resulted in clinically meaningful reductions in $\mathrm{HbA} 1 \mathrm{c}$ compared with GLIM/GLAR, with greater HbA1c reductions in patients with higher baseline HbA1c $(\geq 8.5 \%)$ compared with patients with lower baseline HbA1c $(<8.5 \%)$. Our observation that poorly glycemic-controlled patients with $\mathrm{HbA} 1 \mathrm{c} \geq 8.5 \%$ had greater 


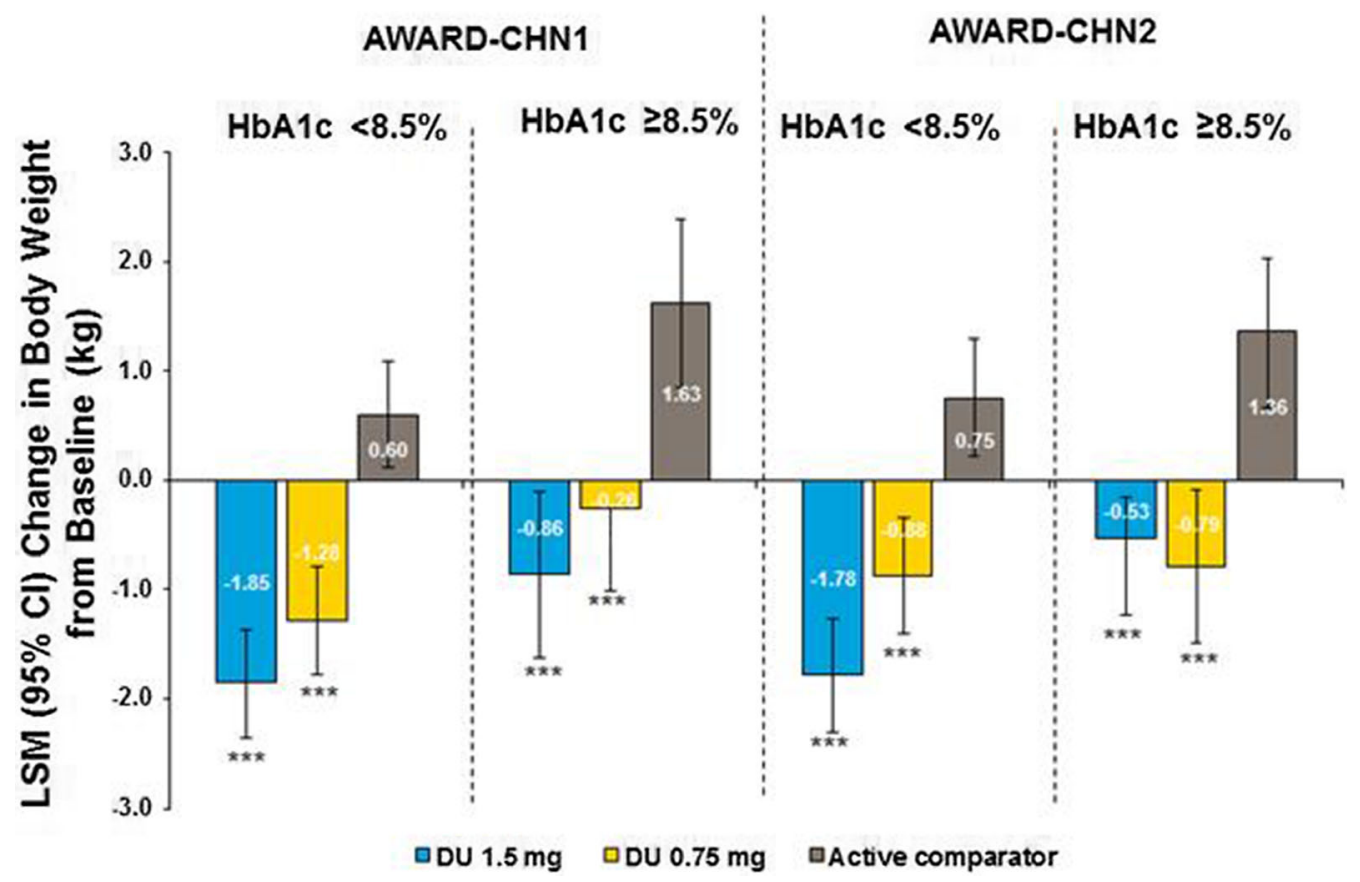

Fig. 4 Change in body weight from baseline to week $26 .{ }^{* * *} p<0.001$, dulaglutide vs. active comparator

HbA1c reductions compared with patients with $\mathrm{HbA} 1 \mathrm{c}<8.5 \%$ is consistent with published data that patients with lower HbA1c tend to experience smaller treatment-induced changes in HbA1c than those with higher HbA1c at baseline [13-15]. This was also reported for liraglutide by Henry et al. [16]. In that post hoc analysis of phase III randomized controlled trials, patients with T2DM were stratified by HbA1c values into five categories ( $\leq 5 \%,>7.5-8.0 \%,>8.0-8.5 \%,>8.5-9.0 \%$, and $>9.0 \%$ ), and reductions in HbA1c levels with liraglutide were generally greater in groups with higher baseline HbA1c [16]. Similar findings were also reported for lixisenatide, with greater reductions in HbA1c in patients with higher baseline HbA1c levels, as shown in a pooled analysis of the lixisenatide GetGoal studies [17]. The observed effect is due to the greater potential for improvement in glycemic control in patients with higher baseline HbA1c. Similar relationships between HbA1c levels and improvements in glycemic control have also been identified in previous reports of metaanalyses of various glucose-lowering therapies [18], meta-analyses of GLP-1RAs other than dulaglutide [19], and global phase III studies of dulaglutide [9]. Similarly, the FBG reductions were consistent with $\mathrm{HbA1c}$ reductions in both dulaglutide doses.

Post hoc analyses of the dulaglutide clinical development program/global AWARD studies (AWARD-1 to -6 and -8 clinical trials), which included mainly Caucasian patients with T2DM, demonstrated significant improvements in glycemic control irrespective of HbA1c levels, with greater HbA1c and FBG reductions in patients with a higher baseline HbA1c [9]. The dulaglutide clinical development program showed that patients with $\mathrm{HbA} 1 \mathrm{c} \geq 8.5 \%$ had greater HbA1c reductions than patients with baseline HbA1c $<8.5 \%$, ( $\geq 8.5 \%$ : LS mean $1.86 \%$ [95\% CI $-1.97,-1.75] ;<8.5 \%$ : LS mean - 1.02\% [95\% CI - 1.12, - 0.93]) [9]. Also, global AWARD studies showed that reductions in FBG were consistent with HbA1c changes. The findings of this post hoc analysis of Chinese data are consistent with the global AWARD studies. In AWARD-CHN1, the HbA1c reductions at 26 weeks with baseline $\mathrm{HbA1c}<$ $8.5 \%$ and $\geq 8.5 \%$, respectively, were dulaglutide $1.5 \mathrm{mg}$ : $-1.1 \%$ and $-2.2 \%$; dulaglutide 


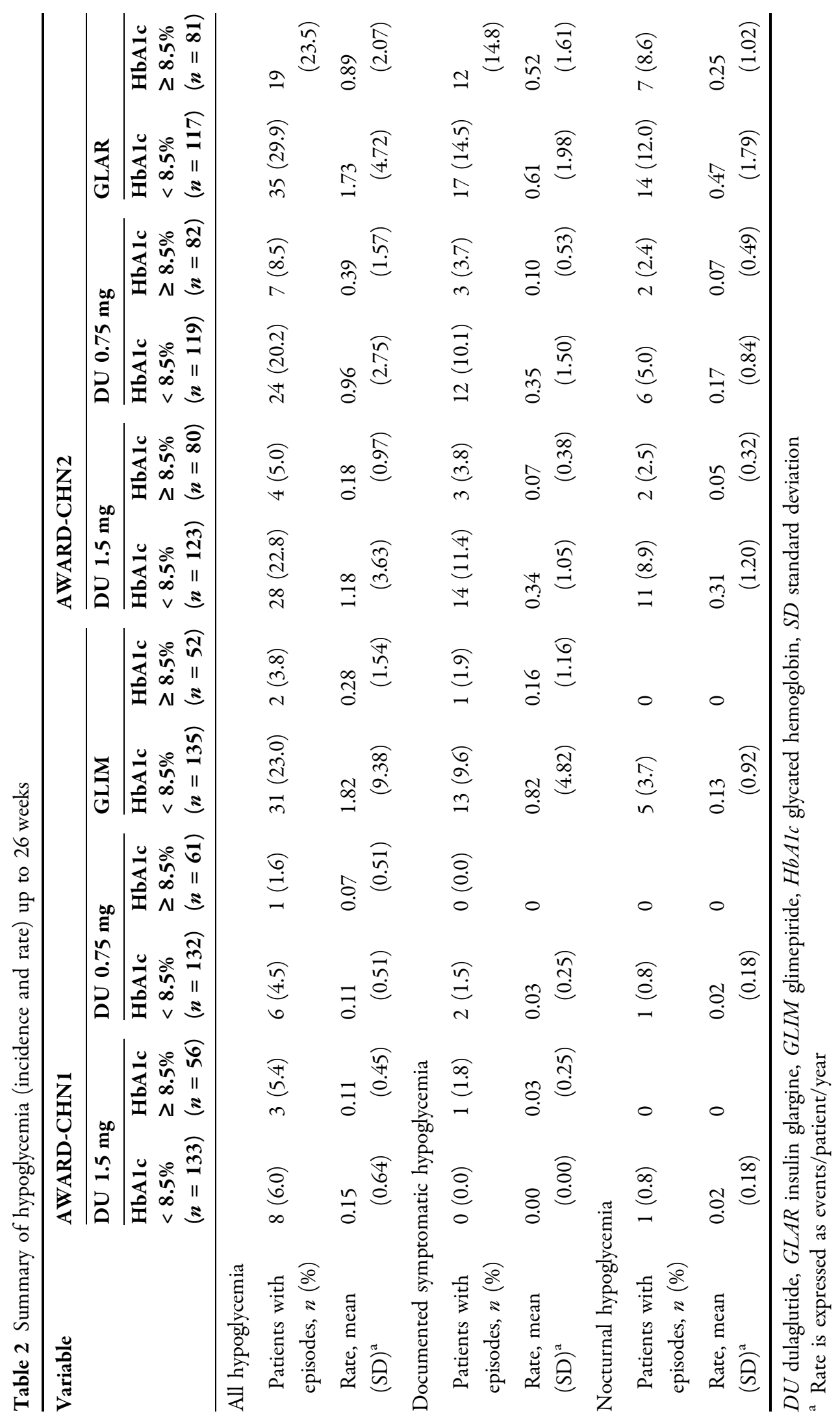




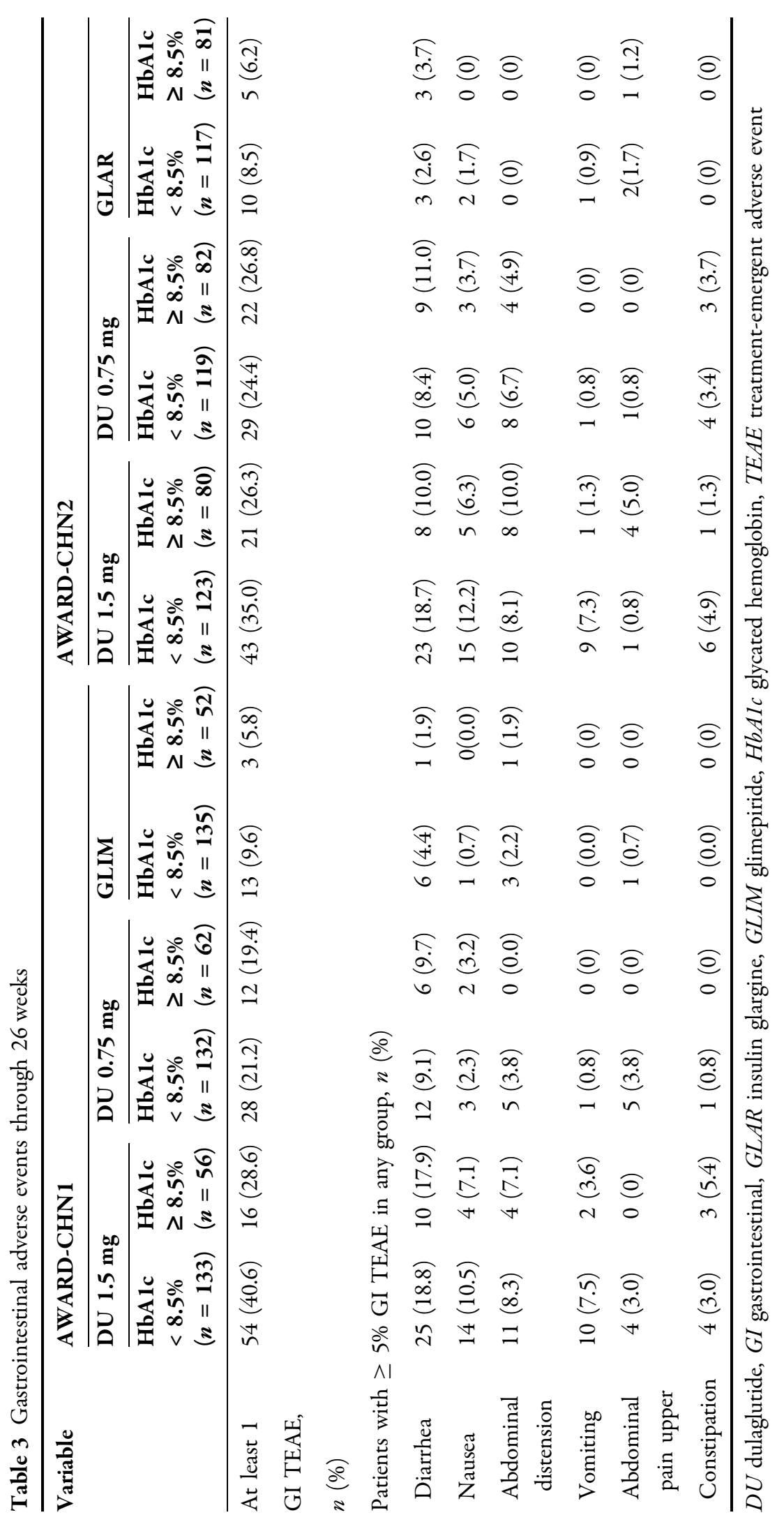


$0.75 \mathrm{mg}: \quad-0.9 \%$ and $-2.0 \%$; glimepiride: $-0.7 \%$ and $-1.4 \%$. In AWARD-CHN2, the HbA1c reductions at 26 weeks with baseline HbA1c $<8.5 \%$ and $\geq 8.5 \%$, respectively, were dulaglutide $1.5 \mathrm{mg}: \quad-1.2 \%$ and $-2.3 \%$; dulaglutide $0.75 \mathrm{mg}: \quad-1.0 \%$ and $-1.7 \%$; insulin glargine: $-0.6 \%$ and $-1.7 \%$. In the dulaglutide and active comparator treatment groups (GLIM/GLAR), the patients with HbA1c $\geq 8.5 \%$ experienced greater reductions in $\mathrm{HbA1c}$ than patients with $\mathrm{HbA1c}<8.5 \%$. Moreover, the present analysis showed dulaglutide 1.5 and $0.75 \mathrm{mg}$ treatments resulted in a greater proportion of patients achieving HbA1c values of less than both $6.5 \%$ and $7 \%$ at 26 weeks compared with active comparators (GLIM/GLAR). Also, in this post hoc analysis, the HbA1c $<8.5 \%$ subgroup had a greater proportion of patients achieving HbA1c values of less than both $6.5 \%$ and $7 \%$ at 26 weeks compared with the $\mathrm{HbA} 1 \mathrm{c} \geq 8.5 \%$ subgroup. This indicates that early initiation of dulaglutide treatment when the HbA1c target is not being met may lead to a better patient outcome.

In this post hoc analysis, dulaglutide-treated patients experienced GI AEs more frequently than active comparator-treated patients; however, all the observed GI AEs were reported as mild to moderate intensity and well tolerated. The incidences of total hypoglycemia, asymptomatic, and nocturnal hypoglycemia were lower with dulaglutide than GLIM/GLAR in both subgroups, with low incidence of hypoglycemia in patients with baseline HbA1c $\geq 8.5 \%$ compared with the patients with baseline $\mathrm{HbA} 1 \mathrm{c}<8.5 \%$. Moreover, incidences of hypoglycemia were higher in insulin glarginetreated patients with lower HbA1c than in patients with higher HbA1c. Overall, in this post hoc analysis, both doses of dulaglutide were well tolerated and the safety profile of dulaglutide was similar to the GLP-1RA class of drugs, suggesting a favorable benefit-to-risk profile for dulaglutide. The findings of the present post hoc analysis are consistent with the findings from global studies (AWARD program) [9] with dulaglutide and with those from other studies with published data for other GLP-1RAs $[20,21]$. The safety and tolerability profile of dulaglutide is similar to that of other agents in the GLP-1RA class $[11,12,20,21]$. The most common side effects are GI related and include nausea, vomiting, diarrhea, and abdominal distension [11, 12]. Also consistent with the GLP-1RA class, GI side effects are mostly mild to moderate, occur early in the course of treatment, and are transient.

The present post hoc analysis has some limitations. Pooled analyses of data of both the included AWARD-CHN studies were not conducted to prevent the confounding effect of the various concomitant background medications used in each study. Additional limitations include: an imbalanced sample size and smaller number of patients with $\mathrm{HbA} 1 \mathrm{c} \geq 8.5 \%$ compared with HbA1c of $\leq 8.5 \%$.

\section{CONCLUSIONS}

In the present post hoc analysis of two studies, dulaglutide demonstrated significantly greater HbA1c reduction with weight loss and lower risk of hypoglycemia than active comparators in Chinese patients with T2DM irrespective of baseline HbA1c, with much greater HbA1c reductions in patients with a higher baseline HbA1c. Dulaglutide was well tolerated, with a safety profile similar to other GLP-1RAs.

\section{ACKNOWLEDGEMENTS}

The authors thank Li Qun $\mathrm{Gu}$, ex-staff from Eli Lilly and Company, for the conception, design, and interpretation of data.

Funding. This study and the journal's Rapid Service Fee were funded and supported by Eli Lilly and Company. All authors had full access to all of the data in this study and take complete responsibility for the integrity of the data and accuracy of the data analysis.

Medical Writing and Editorial Assistance. The authors thank Rakesh Ojha, PhD, from Syneos Health for medical writing support and Tucker Teri, from Syneos Health for editorial support in the preparation of this 
manuscript. Eli Lilly and Company funded writing and editing support.

Authorship. All named authors meet the International Committee of Medical Journal Editors (ICMJE) criteria for authorship for this article, take responsibility for the integrity of the work as a whole, and have given their approval for this version to be published.

Authorship Contributions. Bin Zhang was involved in analysis of data and interpretation of data. Hai $\mathrm{Ya} \mathrm{Wu}$ was involved in conception, design, and interpretation of data. Miao Yu and Xiao Feng Lv were involved in acquisition and interpretation of data. Guo Yue Yuan was involved in acquisition of data.

Disclosures. Bin Zhang and Hai $\mathrm{Ya} \mathrm{Wu}$ are employees of Eli Lilly and Company. Miao Yu, Guo Yue Yuan, and Xiao Feng Lv have nothing to disclose.

Compliance with Ethics Guidelines. Institutional ethics committee approval was obtained from both the studies, and written informed consent was taken from each patient before participation. Both the studies were conducted in consensus with the Declaration of Helsinki, good clinical practice guidelines, and applicable laws and regulations.

Data Availability. The datasets used during and/or analyzed during the current study are available from the corresponding author on reasonable request.

Open Access. This article is licensed under a Creative Commons Attribution-NonCommercial 4.0 International License, which permits any non-commercial use, sharing, adaptation, distribution and reproduction in any medium or format, as long as you give appropriate credit to the original author(s) and the source, provide a link to the Creative Commons licence, and indicate if changes were made. The images or other third party material in this article are included in the article's Creative Commons licence, unless indicated otherwise in a credit line to the material. If material is not included in the article's Creative Commons licence and your intended use is not permitted by statutory regulation or exceeds the permitted use, you will need to obtain permission directly from the copyright holder.To view a copy of this licence, visit http://creativecommons.org/licenses/by$\mathrm{nc} / 4.0 /$

\section{REFERENCES}

1. Olokoba AB, Obateru OA, Olokoba LB. Type 2 diabetes mellitus: a review of current trends. Oman Med J. 2012;27:269-73.

2. American Diabetes Association. Diagnosis and classification of diabetes mellitus. Diabetes Care. 2009;32(Suppl 1):S62-7.

3. Wang L, Gao P, Zhang M, et al. Prevalence and ethnic pattern of diabetes and prediabetes in China in 2013. JAMA. 2017;317:2515-23.

4. Ji L, Feng B, Su Q, et al. The safety and efficacy of initiating insulin therapy in Chinese patients with type 2 diabetes mellitus inadequately controlled with previous oral antidiabetic drugs. Chin J Diabetes. $2011 ; 19: 746-51$.

5. Weng J, Ji L, Jia W, et al. Standards of care for type 2 diabetes in China. Diabetes Metab Res Rev. 2016;32: 442-58.

6. Wang K, Wang K, Chen Y, Hou J, Siddall J. Insulin initiation for type 2 diabetes mellitus in China: physicians' preferred HbA1c level at insulin initiation and patients' observed levels. Value Health. 2016;19:A211.

7. McCall AL. Insulin therapy and hypoglycemia. Endocrinol Metab Clin North Am. 2012;41:57-87.

8. Larger E. Weight gain and insulin treatment. Diabetes Metab. 2005;31:51-6.

9. Gallwitz B, Dagogo-Jack S, Thieu V, et al. Effect of once-weekly dulaglutide on glycated haemoglobin (HbA1c) and fasting blood glucose in patient subpopulations by gender, duration of diabetes and baseline HbA1c. Diabetes Obes Metab. 2018;20: 409-18.

10. Unger JR, Parkin CG. Glucagon-like peptide-1 (GLP1) receptor agonists: differentiating the new medications. Diabetes Ther. 2011;2:29-39.

11. Chen YH, Huang CN, Cho YM. Efficacy and safety of dulaglutide monotherapy compared with 
glimepiride in East-Asian patients with type 2 diabetes in a multicentre, double-blind, randomized, parallel-arm, active comparator, phase III trial. Diabetes Obes Metab. 2018;20:2121-30.

12. Wang W, Nevárez L, Filippova E. Efficacy and safety of once-weekly dulaglutide versus insulin glargine in mainly Asian patients with type 2 diabetes mellitus on metformin and/or a sulphonylurea: a 52-week open-label, randomized phase III trial. Diabetes Obes Metab. 2019;21:234-43.

13. Bloomgarden ZT, Dodis R, Viscoli CM, Holmboe ES, Inzucchi SE. Lower baseline glycemia reduces apparent oral agent glucose lowering efficacy: a meta-regression analysis. Diabetes Care. 2006;29: 2137-9.

14. Phatak HM, Yin DD. Factors associated with the effect-size of thiazolidinedione (TZD) therapy on $\mathrm{HbA}(1 \mathrm{c})$ : a meta-analysis of published randomized clinical trials. Curr Med Res Opin. 2006;22: 2267-78.

15. Chapell R, Gould AL, Alexander CM. Baseline differences in A1C explain apparent differences in efficacy of sitagliptin, rosiglitazone and pioglitazone. Diabetes Obes Metab. 2009;11:1009-16.

16. Henry RR, Buse JB, Sesti G, et al. Efficacy of antihyperglycemic therapies and the influence of baseline hemoglobin $\mathrm{A}(1 \mathrm{C})$ : a meta-analysis of the liraglutide development program. Endocr Pract. 2011;17:906-13.

17. Blonde L, Chava P, Dex T, Lin J, Nikonova EV, Goldenberg RM. Predictors of outcomes in patients with type 2 diabetes in the lixisenatide GetGoal clinical trials. Diabetes Obes Metab. 2017;19: 275-83.

18. DeFronzo RA, Stonehouse AH, Han J, Wintle ME. Relationship of baseline HbA1c and efficacy of current glucose-lowering therapies: a meta-analysis of randomized clinical trials. Diabetes Med. 2010;27:309-17.

19. Seino H, Onishi Y, Naito Y, Komatsu M. Lixisenatide improves glycemic outcomes of Japanese patients with type 2 diabetes: a meta-analysis. Diabetol Metab Syndr. 2016;8:36.

20. Bennett WL, Maruthur NM, Singh S, et al. Comparative effectiveness and safety of medications for type 2 diabetes: an update including new drugs and 2-drug combinations. Ann Intern Med. 2011;154: 602-13.

21. Ostawal A, Mocevic E, Kragh N, Xu W. Clinical effectiveness of liraglutide in type 2 diabetes treatment in the real-world setting: a systematic literature review. Diabetes Ther. 2016;7:411-38. 